\title{
Extended Aharonov-Bohm period analysis of strongly correlated electron systems
}

\author{
Ryotaro Arita $^{1}$, Koichi Kusakabe ${ }^{2}$, Kazuhiko Kuroki ${ }^{1}$, and Hideo Aoki ${ }^{1}$ \\ ${ }^{1}$ Department of Physics, University of Tokyo, Hongo, Tokyo 113, Japan \\ ${ }^{2}$ Institute for Solid State Physics, University of Tokyo, Roppongi, Tokyo 106, Japan
}

(July 27, 2013)

\begin{abstract}
The 'extended Aharonov-Bohm (AB) period' recently proposed by Kusakabe and Aoki [J. Phys. Soc. Jpn 65, 2772 (1996)] is extensively studied numerically for finite size systems of strongly correlated electrons. While the extended $\mathrm{AB}$ period is the system length times the flux quantum for noninteracting systems, we have found the existence of the boundary across which the period is halved or another boundary into an even shorter period on the phase diagram for these models. If we compare this result with the phase diagram predicted from the Tomonaga-Luttinger theory, devised for low-energy physics, the halved period (or shorter periods) has a one-to-one correspondence to the existence of the pairing (phase separation or metal-insulator transition) in these models. We have also found for the $t-J$ model that the extended $\mathrm{AB}$ period does not change across the integrable-nonintegrable boundary despite the totally different level statistics.
\end{abstract}

PACS numbers: 74.20.Mn, 71.10.Hf 


\section{INTRODUCTION}

Aharonov-Bohm (AB) effect is an interesting probe for various phenomena in condensedmatter physics, since this effect explores 'global' responses (over large changes in the gauge flux) rather than linear responses against an infinitesimal change. In this light, it should be especially interesting to look at strongly correlated systems such as the Hubbard model or the $t-J$ model from the AB effect. A natural interest is how the occurrence of superconductivity, if any, should be reflected in the $\mathrm{AB}$ effect. The response of the system to the $\mathrm{AB}$ flux has long been recognized as the 'anomalous flux quantization' as a tool for detecting the Cooper pairing ever since Byers and Yang proposed the idea. Let us pierce an AB flux, $\Phi$, through the torus as shown in Figure.1. In a normal state, the ground-state energy is a periodic function of $\Phi$ with period $\Phi_{0}=h c / e$ with minima at $\Phi / \Phi_{0}=0, \pm 1, \pm 2, \cdots$. In a superconducting phase, new stable states emerge at $\Phi / \Phi_{0}= \pm \frac{1}{2}, \pm \frac{3}{2} \cdots$ due to the pairing, which makes the period $\Phi_{0} / 2$. The method has been applied to several models $⿴$ 日.

The response to an infinitesimal AB flux can also detect the Drude weight $D$ O or the superfluid weight $D_{s} .1011$ The Drude weight is a measure of the ratio of the density of mobile charge carriers to their mass, while the superfluid weight measures the ratio of the superfluid density to mass. For a clean system, we expect $D=D_{s}=0$ for an insulator, $D$ is finite and $D_{s}=0$ for a metal, and $D$ and $D_{s}$ are both finite for a superconductor.

As a test for pairing, however, the conventional anomalous flux quantization can be ambiguous, since the method is, in some cases, not sensitive enough to determine phase boundaries, or because finite-size effects obscure the test. For example, Ferretti et al. shows that both repulsive and attractive Hubbard model behave as a superconductors in the sense of Byers-Yang arguments at $T=0.3$ We can see this in the left corner of Fig.2.

In order to shed a new light, two of the present authors have recently proposed an entirely different method of detecting bound electron states (see Fig.2) 12 The idea stemmed from Sutherland's study for one-dimensional (1D) Heisenberg magnets. 13 He looks at what happens to a many-body state when we twist the boundary condition as 


$$
\phi\left(\cdots, x_{j}+N_{a}, \cdots\right)=e^{i 2 \pi \Phi / \Phi_{0}} \phi\left(\cdots, x_{j}, \cdots\right),
$$

for $N_{a}$-site lattice shifts (or 'boosts') the total momentum of an $N$ particle state. For a noninteracting particles, the boost by $\Phi=\Phi_{0}$ shifts each one-particle momentum by $2 \pi / N_{a}$, so that $\Phi=N_{a} \Phi_{0}$ will bring the set of $k$ points back to the original position.

For an interacting particles we can expect that $\Phi=N_{a} \Phi_{0}$ will also recover the original eigenstate when all of the $N$ particles move individually. By contrast, the response will be different if the particles form $N$-bound states, since bound $N$ particles should have a welldefined center-of-mass momentum, whose response to the AB flux will be $N$ times greater. A twist of $\Phi=N_{a} \Phi_{0} / N$ will then suffice to bring the energy back to the initial value.

For the Heisenberg spin chain Sutherland 3 has in fact confirmed this behavior using the Bethe-ansatz analysis. Kusakabe and Aokit have then proposed that the idea for spin systems may be extended to electron systems, such as the Hubbard model, for detecting bound-electron states. In addition to the Bethe-ansatz analysis for electrons, the method is also numerically implemented in order to reveal the phase diagram of strongly correlated systems in (pseudo-)1D and possibly in 2D systems, where the Bethe ansatz is inapplicable but we can still keep track of the $\Phi$-dependence of the state over the 'extended zone' ( $0 \leq$ $\left.\Phi \leq N_{a} \Phi_{0}\right)$ well beyond the one period $\left(0 \leq \Phi \leq \Phi_{0}\right)$.

Thus this technique is called 'the extended AB period method'. It is conceptually interesting to keep track of the states along the extended zone, since this questions an even more global response of a many-body system than the anomalous flux quantization.

With this method a normal-to-superconducting transition is expected to be characterized as a halving of the extended period. For the 1D Hubbard model, which is an integrable system, this has been shown indeed to be the case 12 The spectral flows have also been analyzed in other integrable systems by Fukui and Kawakami in the context of the exclusion statistics, such as the Haldane-Shastry mode ${ }^{14}$ and the supersymmetric $t$ - $J$ model with $1 / r^{2}$ interaction. 国 On the other hand, the numerical implementation has been applied to non-integrable systems. 12 回 
In the present paper, we investigate extensively the extended $\mathrm{AB}$ method by applying it to one-dimensional (1) $t$ - $J$ model, (2) $t$ - $J$ ladder model, (3) $t-J-J^{\prime}$ model, and (4) the extended Hubbard model to systematically look at the behavior of the extended period.

We address ourselves two fundamental questions:

(i) what is the difference between integrable and non-integrable systems in terms of the extended AB flow,

(ii) can we actually detect normal-superconducting transition or phase separation in such nontrivial models as enumerated above, where a quantum phase transition (at $T=0$ ) is believed to occur at a finite strength of the interaction.

As for the first question, we can naively expect drastically different behaviors for the two classes of systems, since the extended $\mathrm{AB}$ period is dominated by whether the level crossings in the adiabatic flow line turn into anti-crossings, while the quantum adiabatic theorem dictates that the adiabatic evolution of a level is free from level crossings (except for accidental ones) when the system is nonintegrable. We can in fact characterize the avoided crossings in terms of the level statistics (distribution of the spacing of adjacent eigenenergies), which is thought to be universally Gaussian orthogonal (unitary or symplectic depending on the symmetry of the Hamiltonian) for a nonintegrable system while an integrable system has Poisson's distribution as in a noninteracting system. This is because, while levels usually have to repel each other except for accidental degeneracies in the non-integrable case, there exist enough symmetries to characterize a state that allows the levels to cross in the integrable case. This should also apply to many-body systems in integrable or nonintegrable cases. For instance, Di Stasio and Zotos ${ }^{17}$ have obtained level statistics for spinless fermions in one dimension with nearest and next-nearest-neighbor interaction. Thus it is intriguing whether the extended spectral flow analysis, which has been shown to be useful in integrable systems from the Bethe-Ansatz analysis, could be applied to non-integrable systems as well. We find here that the spectral flow is smooth in solvable cases as opposed to wiggly flows in nonintegrable cases, but that its period does not change across the integrable-nonintegrable boundary. 
For the question (ii) we conclude here that the extended $\mathrm{AB}$ method can indeed detect a symptom of changes in characters of quantum states even for non-integrable system. For the 1D $t$ - $J$ model, the phase diagram has been obtained by Ogata et al. 1 by combining the Tomonaga-Luttinger (TL) theory 21 and the Lanczos diagonalization of finite systems.

With the same method, Ogata et al.2. 2 obtained the phase diagram of 1D $t-J-J^{\prime}$ model, and Sano and Ōnoㄹ, and independently Kuroki et a 4 , obtained the phase diagram of extended Hubbard model. For the $t-J$ ladder, several authors have obtained the phase diagram using the TL theory assuming that the ladder system may be described by a 1D effective theory.25 20 The phase diagrams obtained from the extended $A B$ results are qualitatively consistent with those predicted by TL theory.

The organization of the paper is as follows. In section 2 we describe the numerical implementation for keeping track of the extended flow. We shall then discuss the result for the 1D $t$ - $J$ model in section 3, where we perform the Bethe-ansatz analysis to discuss the origin of the extended periodicity. Specifically, we discuss the behavior of the extended AB period in the non-integrable region from a numerical analysis. Although the period does not change across the integrable-nonintegrable boundary, their difference does appear in line shapes of the spectral flow. We discuss the results for the $t-J$ ladder (Section 4), 1D $t-J-J^{\prime}$ model (Section 5) and extended Hubbard model (Section 6).

\section{NUMERICAL ALGORITHM}

In general we want to treat non-integrable systems. For instance the $t$ - $J$ model is nonintegrable even in $1 \mathrm{D}$ except at special points, i.e., $J=0(U=\infty$ Hubbard model $)$ or $J=2 t$ (the supersymmetric $t-J$ model). Thus we must numerically implement the method to obtain the extended $\mathrm{AB}$ period. Here we recapitulate the numerical algorithm proposed in Ref.12, which consists of successive estimations of the energy and wave function.

1. First solve $H(\Phi=0) \phi=E(\Phi=0) \phi$ by a conventional diagonalization technique, (e.g., the Lanczos method). 
2. Predict $\tilde{E}_{n=0}(\Phi+\Delta \Phi)$ by $\tilde{E}_{n=0} \equiv\langle\phi(\Phi)|H(\Phi+\Delta \Phi)| \phi(\Phi)\rangle$.

3. Determine $\phi_{n+1}(\Phi+\Delta \Phi)$ by the power method,

$$
\left[\frac{1}{H(\Phi+\Delta \Phi)-\tilde{E}_{n}}\right]^{m} \phi(\Phi) \rightarrow \phi_{n+1}(\Phi+\Delta \Phi)
$$

4. Determine $\tilde{E}_{n+1}(\Phi+\Delta \Phi)$ by $H(\Phi+\Delta \Phi) \phi_{n+1}(\Phi+\Delta \Phi)=\tilde{E}_{n+1}(\Phi+\Delta \Phi) \phi_{n+1}(\Phi+\Delta \Phi)$.

5. If a convergence criterion, $\left|\tilde{E}_{n+1}(\Phi+\Delta \Phi)-\tilde{E}_{n}(\Phi+\Delta \Phi)\right| \leq \varepsilon$ is met with typically $\varepsilon \sim 10^{-8}$, then increase $\Phi$ and go to 2 . Otherwise, increase $n$ and go to 3 .

Usually the energy converges faster than the wavefunction, so that the above algorithm works well. Typically, a few tens of $m$ and $n$ give a convergence.

Around level crossings a care must be taken. There are two types of level crossings. One type occurs between levels that have different symmetries. In this case we can readily go across the crossing, since our method looks at the wavefunction, which is a representation of all of the symmetries of the system. The second type occurs at a critical point, at which a gap opens in the spectral flow. Thus we must question how a crossing turns into an anti-crossing from crossing at a critical point. We can systematically keep track of this by varying interaction parameters around the critical point.

\section{1D $T-J$ MODEL}

We first analyze the extended $\mathrm{AB}$ period of the $1 \mathrm{D} t-J$ ring, where the main interest is whether a change in the period detects a symptom in characters of quantum states. The phase diagram of this model has been investigated by the Tomonaga-Luttinger theory combined with numerical results, 1 with which we can compare our result.

The $t-J$ model belongs to a class of the strongly correlated model in which the electron repulsion is assumed to be infinite so that no double occupancies of electrons are allowed. The Hamiltonian is given by 


$$
H=-t \sum_{\langle i, j\rangle \sigma}\left(\tilde{c}_{i \sigma}^{\dagger} \tilde{c}_{j \sigma}+\text { H.c. }\right)+J \sum_{\langle i, j\rangle}\left(\mathbf{S}_{i} \cdot \mathbf{S}_{j}-\frac{1}{4} n_{i} n_{j}\right)
$$

where $\tilde{c}_{i \sigma}^{\dagger}$ creates at the $i$-th site with spin $\sigma$ and $S_{i}$ is the spin operator defined by $S_{i}=$ $\frac{1}{2} \tilde{c}_{i \sigma}^{\dagger} \vec{\sigma}_{\sigma \sigma^{\prime}} \tilde{c}_{i \sigma^{\prime}}$ with the Pauli matrix $\vec{\sigma}_{\sigma \sigma^{\prime}}$. Hereafter we set $t=1$ and the phase diagram will be parameterized by $J$ and the density of electrons.

Figure 3, 4, 5 shows the numerical result for the spectral flow of the $1 \mathrm{D} t-J$ ring with various values of $J$. Here each system has 4 electrons, while the size of the system is varied from 8 (Fig.3), 10 (Fig.(4) to 12 (Fig.5). We can immediately notice three features28:

- The extended period, which is originally $N_{a} \Phi_{0}$ at $J=0$, is abruptly halved as soon as $J \neq 0$ is turned on.

- At a certain $J_{C}>2$, whose value depends on the band filling, a level anticrossing appears in the flow line. For 8- and 12-site systems, this makes the period further halved into a $1 / 4$ period compared to that for $J=0$. In the case of a $10(\neq 4, \bmod 4)$ site system, the appearance of the $1 / 4$ period is less perfect.

- Solvable points $(J=0,2)$ are distinguished by smooth, cosine-like flow lines. This sharply contrasts with the flow lines in the non-integrable case, where the lines wiggle due to ubiquitous level anticrossings. Despite these, the extended AB period does remain the same across the integrable-nonintegrable boundary, which is explicitly shown by the fact that the curvature of the anticrossing shows no singularities for all values of $J$ considered here.

In the following sections, we explore why these features should arise. We divide the regions into exactly solvable points $(J=0, J=2$ ), and other regions (normal: $0<J<2$; possibly superconducting or phase-separated: $2<J$ ). 


\section{A. $J=0$}

Since the $t-J$ model with $J=0$ is equivalent to the $U=\infty$ Hubbard model, let us start with the 1D Hubbard model. Consider an $N_{a}$-site ring (with $N_{a}$ even for simplicity) containing $N$ electrons. The Hamiltonian is given by

$$
H=-t \sum_{i \sigma}\left(c_{i \sigma}^{\dagger} c_{i+1 \sigma}+\text { H.c. }\right)+U \sum_{i} n_{i \uparrow} n_{i \downarrow}
$$

where $c_{i \sigma}\left(c_{i \sigma}^{\dagger}\right)$ annihilates (creates) an electron at the $i$-th site with spin $\sigma, n_{i \sigma} \equiv c_{i \sigma}^{\dagger} c_{i \sigma}, U$ the on-site interaction, and $t$ is the transfer energy taken to be the unit of energy hereafter.

We can analyze the 1D Hubbard model in terms of the exact Bethe-ansatz (BA) solution due to Lieb and Wu.29 If we thread a magnetic flux $\Phi$ through the ring, the BA equations become日

$$
\begin{aligned}
& e^{i k_{j} N_{a}}=e^{i 2 \pi \Phi / \Phi_{0}} \prod_{\alpha=1}^{M} e\left(\frac{4\left(\sin k_{j}-\lambda_{\alpha}\right)}{U}\right) \\
& \prod_{j=1}^{N} e\left(\frac{4\left(\lambda_{\alpha}-\sin k_{j}\right)}{U}\right)=\prod_{\beta=1}^{M} e\left(\frac{2\left(\lambda_{\alpha}-\lambda_{\beta}\right)}{U}\right) .
\end{aligned}
$$

Here $M(\leq N / 2)$ is the number of down-spin electrons, $e(x) \equiv(x+i) /(x-i)$, and $k_{j}\left(\lambda_{\alpha}\right)$ is the charge (spin) rapidities, which are roughly a quasimomenta for the charge (spin).

When $U=\infty$, the charge part of the BA equation (eq.(3.1)) becomes 30

$$
\begin{aligned}
k_{j} N_{a} & =2 \pi I_{j}+2 \pi \Phi / \Phi_{0}+P_{H}, \\
P_{H} & =\sum_{\alpha=1}^{M} 2 \tan ^{-1}\left(2 \Lambda_{\alpha}\right),
\end{aligned}
$$

with $\Lambda_{\alpha}=2 \lambda_{\alpha} / U$. This shows that in the strong-interaction limit the allowed values of $k_{j}$ 's are quantized in units of $2 \pi / N_{a}$. In other words, charge degrees of freedom act like spinless fermions. Hence the flow line has a periodicity of $N_{a} \Phi_{0}$.

\section{B. $J=2$, the supersymmetric point}

$J=2$ is special in that the model is integrable with the Bethe-ansatz solution, which we first briefly summarize. Lai 31 and later Schlottmann, 32 proved that solving the problem 
at $J=2$ reduces to solving a set of $(N+M)$-coupled algebraic equations for $N$ charge rapidities $v_{j}$ and $M$ spin rapidities $\Lambda_{\alpha}$, i.e.,

$$
\begin{array}{r}
\exp \left(i k_{j} N_{a}\right)=\prod_{\beta=1}^{M} \frac{v_{j}-\Lambda_{\beta}+i / 2}{v_{j}-\Lambda_{\beta}-i / 2}, \\
\prod_{j=1}^{N} \frac{v_{j}-\Lambda_{\alpha}+i / 2}{v_{j}-\Lambda_{\alpha}-i / 2}=-\prod_{\beta=1}^{M} \frac{\Lambda_{\beta}-\Lambda_{\alpha}+i}{\Lambda_{\beta}-\Lambda_{\alpha}-i},
\end{array}
$$

with $v_{j}=\cot \left(k_{j} / 2\right) / 2$. The ground state is given by a set of complex charge rapidities (two-strings) and real spin rapidities, where a spin rapidity lies at the center of each two string.

Sutherland 33 found an alternative way of solving the problem. There we treat the holes and down-spin electrons as dynamical objects in a background of up-spin electrons. In this formalism, BA equations become

$$
\begin{aligned}
\exp \left(i k_{\alpha} N_{a}\right) & =-\prod_{j=1}^{M_{2}} \frac{v_{\alpha}-w_{j}+i / 2}{v_{\alpha}-w_{j}-i / 2} \prod_{\beta=1}^{M_{1}} \frac{v_{\alpha}-v_{\beta}-i}{v_{\alpha}-v_{\beta}+i}, \\
1 & =\prod_{\beta=1}^{M_{1}} \frac{w_{j}-v_{\beta}-i / 2}{w_{j}-v_{\beta}+i / 2} .
\end{aligned}
$$

Here $v_{\alpha}=\tan \left(k_{\alpha} / 2\right) / 2$ with $\alpha=1, \cdots, M_{1}=N_{h}+M$ involves both spin and charge degrees of freedom, while $w_{j}$ is the hole rapidity that represents charge degrees of freedom with $j=1, \cdots, M_{2}=N_{h}$ with $N_{h}$ being the number of holes. A particle-hole transformation connects Sutherland's representation to that of Lai or Schlottmann.34.35

Sutherland's formalism is more appealing for numerical calculations, since (i) the ground state involves only real roots, and (ii) we can directly investigate the behavior of the charge rapidities. So we follow Sutherland. If we take the logarithm of Sutherland's BA equations we have

$$
\begin{aligned}
N_{a} \theta\left(2 v_{\alpha}\right) & =2 \pi J_{\alpha}+\sum_{\beta=1}^{M_{1}} \theta\left(v_{\alpha}-v_{\beta}\right)-\sum_{j=1}^{M_{2}} \theta\left(2\left(v_{\alpha}-w_{j}\right)\right), \\
2 \pi I_{j} & =\sum_{\beta=1}^{M_{1}} \theta\left(2\left(w_{j}-v_{\beta}\right)\right),
\end{aligned}
$$

with $\theta(x) \equiv 2 \tan ^{-1}(x) . J_{\alpha}$ is an integer (half odd integer) when $M_{1}-M_{2}+1$ is even (odd), while $I_{j}$ is an integer (half odd integer) if $M_{1}$ is even (odd). Here we assume $I_{1}<I_{2}<\cdots<$ 
$I_{M_{2}}, J_{1}<J_{2}<\cdots<J_{M_{1}}$. For the ground state, we must choose $\left|J_{\alpha}\right|$ 's and $\left|I_{j}\right|$ 's as close to 0 as possible.

If we thread a magnetic flux $\Phi$, the left hand side of the charge part of the BA equation becomes

$$
2 \pi \tilde{I}_{j} \equiv 2 \pi I_{j}-2 \pi \Phi / \Phi_{0}
$$

In Fig.6 we show how the rapidities evolve with $\Phi$ for the ground state. We can see that the rapidities change with a characteristic manner with a periodicity of $N_{a} \Phi_{0} / 2$. Conspicuously, the charge rapidities, $w_{j}$ 's, respond to the magnetic flux in pairs. The pair diverges to $\pm \infty$ at some points, around which the pair sandwiches other rapidity $v_{\alpha}$. Since each pair of charge rapidities exchange their places as they cross from $-\infty$ to $+\infty$, the charge rapidities are shuffled like

$$
\left\{w_{1}, w_{2}, w_{3}, w_{4}, \cdots\right\} \rightarrow\left\{w_{2}, w_{1}, w_{4}, w_{3}, \cdots\right\}
$$

as one extended period ( $\left.\Phi=0 \rightarrow \Phi=N_{a} \Phi_{0} / 2\right)$ is accomplished. The shuffle is similar to the case of the small $J$ limit, as we shall see in the next section. We now look more closely at the BA equations.

\section{The charge part of the BA equation}

As $2 \pi \tilde{I}_{j}$ becomes smaller than $-M_{1} \pi, w_{1}$ and $w_{2}$ vary from $-\infty$ to $\infty$. At the same time, $v_{1}$ varies from $-\infty$ to $\infty$. In order to fix the range of $\tan ^{-1}$, we redefine the quantum numbers $I_{j}$ so that $\left\{\tilde{I}_{1}, \tilde{I}_{2}, \cdots, \tilde{I}_{M_{2}}\right\}=\left\{-M_{1} / 2,-M_{1} / 2+1,-M_{1} / 2+2, \cdots,-M_{1} / 2+M_{2}-1\right\}$ becomes $\left\{M_{1} / 2-1, M_{1} / 2,-M_{1} / 2+1, \cdots,-M_{1} / 2+M_{2}-2\right\}$. When this process is repeated, $\left\{\tilde{I}_{j}\right\}$ comes back to $\left\{I_{j}\right\}$ when $\Phi=N_{a} \Phi_{0} / 2$.

\section{The spin part of the BA equation}

When $v_{1}$ varies from $-\infty$ to $\infty$, the quantum numbers will be redefined so that $\left\{J_{1}, J_{2}, \cdots, J_{M_{1}}\right\}$ becomes $\left\{J_{1}+M_{1}-1, J_{2}-1, J_{3}-1, \cdots, J_{M_{1}}-1\right\}$ for the present choice 
of the Riemann plane. As a set, they do not change.

Accordingly the BA equations have a periodicity of $N_{a} \Phi_{0} / 2$, so that the rapidities return to the original values as a set when $\Phi=N_{a} \Phi_{0} / 2$. Note that in this case, the spectral flow corresponds to a trajectory of the holon-antiholon excitations considered by Bares et al. 36

\section{C. $0<J<2$}

Remarkably, above halving of the extended $\mathrm{AB}$ period for a sizeable $J=2$ is seen to emerge already for an infinitesimal $J$ from the numerical result. In other words, the $t-J$ model with a finite $J$ and the Hubbard model have different extended AB periods. Here we show why an infinitesimal $J$ is enough to halve the extended AB period. For a small $J / t$ we can treat the $J$-term perturbatively. One can start from the $N$-particle state expanded as

$$
|F\rangle=\sum_{x_{1}} \cdots \sum_{x_{N}} \sum_{\sigma_{1}} \cdots \sum_{\sigma_{N}} f_{\sigma_{1} \cdots \sigma_{N}}\left(x_{1} \cdots x_{N}\right) \prod c_{x_{1} \sigma_{1}}^{\dagger} \cdots c_{x_{N} \sigma_{N}}^{\dagger}|0\rangle
$$

For $J=0$, it is known 30 that the coefficient $f$ factorizes into charge and spin parts as

$$
f_{\sigma_{1} \cdots \sigma_{N}}\left(x_{1} \cdots x_{N}\right)=(-1)^{Q} \operatorname{det}\left[\exp \left(i k_{i} x_{Q j}\right)\right] \Phi\left(y_{1} \cdots y_{M}\right)
$$

where $x_{Q 1}<x_{Q 2}<x_{Q 3} \cdots<x_{Q N}$ are the coordinates of all the electrons with $Q$ being a permutation and $k_{j}$ the momentum of a free spinless electron, while $y_{1}<y_{2} \cdots<y_{M}$ are the coordinates of down spins in the spin configuration. The determinant depends only on the positions of particles $\left(x_{Q 1}, \cdots, x_{Q N}\right)$ and not on the positions of down spins $\left(y_{1}, \cdots, y_{M}\right)$. The spin part $\Phi\left(y_{1} \cdots y_{M}\right)$ is identical to the wavefunction of the $1 \mathrm{D}$ Heisenberg ring of length $N$.

When a small $J$ is turned on, characteristic change occurs at a special point in the flow, i.e., highly degenerate points. Effect of $J$ first appears as a lifting of this degeneracy, which can be examined by the first-order degenerate perturbation, as is done 2 for the case of the 1D Hubbard model for $U \sim 0$. The effective Hamiltonian in the first-order perturbation becomes 22 


$$
H_{\mathrm{eff}}=-J\left\langle n\left|\sum_{i} n_{i} n_{i+1}\right| m\right\rangle_{S F} \sum_{j} \mathbf{S}_{j} \cdot \mathbf{S}_{j+1}
$$

where $n_{i}=c_{i}^{\dagger} c_{i}$ is the number operator of spinless fermions, and $\langle n|\cdots| m\rangle_{S F}$ is the matrix element between wave functions of non-interacting spinless fermions having the same energy. The summation over $j$ is taken over the compressed spin chain (ignoring the hole sites). If we Fourier transform the first part of the effective Hamiltonian as

$$
-J \sum_{i} c_{i}^{\dagger} c_{i} c_{i+1}^{\dagger} c_{i+1}=-\frac{J}{N_{a}} \sum_{k, k^{\prime}, q} e^{\mathrm{i} q} c_{k-q}^{\dagger} c_{k} c_{k^{\prime}+q}^{\dagger} c_{k^{\prime}}
$$

we can see that when $J$ is turned on, the degenerate states having $\left\{\cdots, k, \cdots, k^{\prime}, \cdots\right\}$ can mix to give an anti-crossing with the states having $\left\{\cdots, k-q, \cdots, k^{\prime}+q, \cdots\right\}$. For these states to have the same energy, we have $\left(k+k^{\prime}\right) / 2 \equiv \pm \pi / 2(\bmod 2 \pi)=\pi / 2$ or $3 \pi / 2$.

We can compare in Figs. $3 \sim 5$ the numerical result for $J=0.1$ with that for $J=0$ obtained by the Bethe-Ansatz assuming $q=\pi$. It can be seen that the spectral flow at $J=0.1$ delineates the lower envelope of the $J=0$ flows, suggesting that we have indeed $q=\pi$. For example, let us consider the case of 4 electrons in 12 sites (Fig.5). When $J$ is turned on, the rapidities $\left\{k_{j}\right\}$ change as

$$
\left\{\frac{\pi}{12}, \frac{3 \pi}{12}, \frac{5 \pi}{12}, \frac{7 \pi}{12}\right\} \rightarrow\left\{\frac{\pi}{12}, \frac{3 \pi}{12}, \frac{5 \pi}{12}-\pi=\frac{-7 \pi}{12}, \frac{7 \pi}{12}+\pi \equiv \frac{-5 \pi}{12}\right\}
$$

at $\Phi=2.5 \Phi_{0}$, and

$$
\left\{\frac{5 \pi}{12}, \frac{7 \pi}{12}, \frac{-3 \pi}{12}, \frac{-\pi}{12}\right\} \rightarrow\left\{\frac{5 \pi}{12}-\pi=\frac{-7 \pi}{12}, \frac{7 \pi}{12}+\pi \equiv \frac{-5 \pi}{12}, \frac{-3 \pi}{12}, \frac{-\pi}{12}\right\}
$$

at $\Phi=4.5 \Phi_{0}$.

We can indeed give a perturbational argument why the umklapp $(q=\pi)$ is selected. For $J=0$, the state $\left|\frac{\pi}{12}, \frac{3 \pi}{12}, \frac{5 \pi}{12}, \frac{7 \pi}{12}\right\rangle \equiv|0\rangle$ is degenerate at $\Phi=2.5 \Phi_{0}$ with the states $\left|\frac{\pi}{12}, \frac{3 \pi}{12}, \frac{5 \pi}{12}-q, \frac{7 \pi}{12}+q\right\rangle \equiv|q\rangle$, where $q$ is one of vectors, $\frac{3 \pi}{6}, \frac{4 \pi}{6}, \frac{5 \pi}{6}, \frac{6 \pi}{6}, \frac{-4 \pi}{6}$ or $\frac{-5 \pi}{6}$.

The matrix for $\sum n_{i} n_{i+1}$ appearing in eq.([3) spanned by these states are 


\begin{tabular}{|c|c|c|c|c|c|c|c|}
\hline & $q=0$ & $6 \pi / 6$ & $5 \pi / 6$ & $4 \pi / 6$ & $6 \pi / 6$ & $-4 \pi / 6$ & $-5 \pi / 6$ \\
\hline$q=0$ & 1 & $a^{-6}$ & $a^{-5}$ & $a^{-4}$ & $a^{-3}$ & $a^{4}$ & $a^{5}$ \\
\hline $6 \pi / 6$ & $a^{6}$ & 1 & $a$ & $a^{2}$ & $a^{3}$ & $a^{10}$ & $a^{11}$ \\
\hline $5 \pi / 6$ & $a^{5}$ & $a^{-1}$ & 1 & $a$ & $a^{2}$ & $a^{9}$ & $a^{10}$ \\
\hline $4 \pi / 6$ & $a^{4}$ & $a^{-2}$ & $a^{-1}$ & 1 & $a$ & $a^{8}$ & $a^{9}$ \\
\hline $3 \pi / 6$ & $a^{3}$ & $a^{-3}$ & $a^{-2}$ & $a^{-1}$ & 1 & $a^{7}$ & $a^{8}$ \\
\hline$-4 \pi / 6$ & $a^{-4}$ & $a^{-10}$ & $a^{-9}$ & $a^{-8}$ & $a^{-7}$ & 1 & $a$ \\
\hline$-5 \pi / 6$ & $a^{-5}$ & $a^{-11}$ & $a^{-10}$ & $a^{-9}$ & $a^{-8}$ & $a^{-1}$ & 1 \\
\hline
\end{tabular}

where $a \equiv \exp (i \pi / 6)$. This matrix has one nonzero eigenvalue, 7 , with the eigenvector $|m\rangle \equiv\left(1, a^{6}, a^{5}, a^{4}, a^{3}, a^{-4}, a^{-5}\right)$, while all the other eigenvalues are zero. Hence, when a small $-J \sum_{i} n_{i} n_{i+1}$ is turned on, the degeneracy is partially removed with a single level peeled off (Fig.7). For the flows to be connected continuously, the state $|0\rangle$ must transform as $|0\rangle \rightarrow|m\rangle \rightarrow|q=\pi\rangle$, which exactly implies $q=\pi$. The above argument holds for any numbers of electrons and sites. Interestingly, this behavior is similar to that for the attractive Hubbard model, in which $q=\pi$ holds as well.

The motion or the 'selection rule' for the rapidities can be seen more directly in Fig.8, where two $k$ 's pair off and respond to $\Phi$ hand in hand. We can interpret this as an effect of $J$ working as an attractive interaction. As a consequence, a finite $J$ halves the period of the spectral flow. Namely, the $k$ 's for the ground state change from $\left\{k_{1}, k_{2}, k_{3}, k_{4}, \cdots,\right\}$ to $\left\{k_{2}, k_{1}, k_{4}, k_{3}, \cdots,\right\}$ for $\Phi=0 \rightarrow N_{a} \Phi_{0} / 2$.

This behavior is reminiscent of the charge rapidities in Fig.6, which can be defined when the system is integrable at $J=2$, the supersymmetric case. For $J=2$, Bares et al 36 have termed the phase as 'weakly bound electron pairs' having antiparallel spins because charge rapidities by Lai's representation form two strings. In terms of the extended AB period, the numerical result shows that the period is the same over the whole region of $0<J<J_{C}$. In this sense the 'weakly bound pairs' region extends over $0<J<J_{C}$. 


\section{D. $J>2$}

The present result, Figs. $\sim 5$, exhibits a period halving at a critical $J_{C}(>2)$. In order to determine $J_{C}$, we have evaluated the energy gap $\Delta$ in the following way. First, we identify, with a linear extrapolation, the point $E_{0}$ at which levels would cross if the level repulsion were absent. The energy gap is then estimated as $\Delta=E_{0}-E_{1}$, where $E_{1}$ is the energy of the repelled level. We can next plot $\Delta$ as a function of $J$ and determine $J_{C}$ as $\Delta\left(J_{C}\right)=0$ by extrapolation. The results are $J_{C} \simeq 2.30$ for 4 electrons in 8 sites, $J_{C} \simeq 2.22$ for 4 electrons in 10 sites, $J_{C} \simeq 2.14$ for 4 electrons in 12 sites.

According to the phase diagram obtained by Ogata et al, 18 the superconducting correlation function becomes dominant $\left(K_{\rho}>1\right)$ in the region $J>J_{c}$, where $J_{c}>2$ is a function of the band filling. The phase boundary is identified as the trajectory of $K_{\rho}=1$ in terms of the exponent $K_{\rho}$ that characterizes the TL liquid. The boundary between superconducting and phase separated regions is more or less pararell to the trajectory of $K_{\rho}=1$.

Figure 9 superposes the period halving points on the phase diagram (trajectory of $K_{\rho}=$ 1) the TL result 18 . We can see the halving points fall on a line near the trajectory of $K_{\rho}=1$, which is pararell to the boundary line between superconducting and phase separated regions.

\section{IV. $T$ - $J$ LADDER MODEL}

We now turn to the $t$ - $J$ ladder model (for a review of the ladder, see, e.g., Ref.37). The original motivation to introduce ladders came from an expectation that a gap in spin excitations may be formed in such systems, leading possibly superconductivity when carriers are doped. The importance of the spin gap has in turn conceived for the high- $T_{c}$ cuprates. Specifically, the $t-J$ ladder has been predicted to have superconductivity associated with a spin gap when there are even number of chains $33-40$. The interests are heightened when a class of cuprates are found to possess the ladder structure in the $\mathrm{Cu}-\mathrm{O}$ network. $41-43$ Very re-

cently, a superconductivity has been detected in $(\mathrm{Ca}, \mathrm{Sr}){ }_{14} \mathrm{Cu}_{24} \mathrm{O}_{41}$ under high pressures.44, 45 
This has kicked off numerical works with the exact diagonalization or the densitymatrix renormalization group (DMRG) method 384 427.49 Since the system is quasi-1D, the Tomonaga-Luttinger (TL) liquid theory has also been applied.2527 Some results have predicted existence of a superconducting phase, in which a spin gap is also shown to survive the doping near the half filling 46 However, the ladder is not purely $1 \mathrm{D}$, the validity of the TL approach has to be justified. Hence, another method detecting the pairing is desirable.

Thus it is most intriguing how the extended AB period test predict the transition for the $t$ - $J$ ladder. Kusakabe and Aoki 22 obtained a preliminary result that the extended AB period changes around the phase boundary predicted by Sano.26 We discuss the extended AB period of this model in more detail.

The $t$ - $J$ ladder is defined, in standard notations, by the Hamiltonian,

$$
\begin{aligned}
H & =-t \sum_{i, \alpha, \sigma} P_{G}\left(c_{i, \alpha, \sigma}^{\dagger} c_{i+1, \alpha, \sigma}+\text { h.c. }\right) P_{G}-t_{\perp} \sum_{i, \sigma} P_{G}\left(c_{i, 1, \sigma}^{\dagger} c_{i, 2, \sigma}+\text { h.c. }\right) P_{G} \\
& +J \sum_{i, \alpha}\left(\mathbf{S}_{i, \alpha} \cdot \mathbf{S}_{i+1, \alpha}-\frac{1}{4} n_{i+1, \alpha} n_{i, \alpha}\right) \\
& +J_{\perp} \sum_{i}\left(\mathbf{S}_{i, 1} \cdot \mathbf{S}_{i, 2}-\frac{1}{4} n_{i, 1} n_{i, 2}\right)
\end{aligned}
$$

where $\alpha$ labels two legs of the ladder, while $i$ labeling the rung runs from 1 to $N_{a}$. Doubly occupied sites are totally excluded by the Gutzwiller projection $P_{G}$ as in the single $t-J$ model.

Here we take the same intra- and inter-chain electron transfer, $t=t_{\perp}$, and the same intraand inter-chain superexchange interaction, $J=J_{\perp}$, for simplicity. The AB flux threaded to the ladder wound along the length introduces a Peierls phase as

$$
c_{i, \alpha, \sigma}^{\dagger} c_{i+1, \alpha, \sigma} \rightarrow \exp \left(i \frac{2 \pi \Phi}{N_{a} \Phi_{0}}\right) c_{i, \alpha, \sigma}^{\dagger} c_{i+1, \alpha, \sigma} .
$$

We have numerically obtained the extended spectral flow for finite $t$ - $J$ ladders. Results depends on the number of electrons. 


\section{A. Two-Electron Systems}

Result for two electrons in an $N_{a} \times 2=8 \times 2$-site system is shown in Fig.10 We look at the $J$-dependence of the flow of the ground state, starting from the $t$ model (the system with no superexchange interactions, $J=0$, but the double occupancies still excluded). For $J=0$ the flow line has a periodicity of $N_{a} \Phi_{0}$, which is the period expected for individually moving particles. Remarkably, a small $J(=0.4 t)$ is enough to halve the periodicity. This behavior, which is common to two-electron systems on $2 n \times 2$-site ladder ( $n$ : an integer), is caused again by a pair of level anticrossings in the flow. The change in the period from $N_{a} \Phi_{0}$ to $N_{a} \Phi_{0} / 2$ is similar to the result for the single-chain $t-J$ model (Section 3), in which the period is also halved for an infinitesimal $J$. For general numbers of electrons in a ladder, however, the period $N_{a} \Phi_{0}$ is not always observed even for $J=0$. For some numbers of electrons, the halved period, $N_{a} \Phi_{0} / 2$, is often detected (see below) for the $t$ ladder, although $t$ model has no explicit source of attractions between electrons.

\section{B. Four-Electron Systems}

We move on to the spectral flows for four electrons for an $8 \times 2$-site system (1/8-filling) in Fig.11 for various values of $J$. We can see that there exist a pair of level anti-crossings at $J_{C}(\simeq 2.6 t)$, which reduces the extended period into $N_{a} \Phi_{0} / 4$.

For a $6 \times 2$-site system (1/6-filling) in Fig.12, a similar appearance of a pair of level anticrossings occurs at $J_{C} \simeq 2.04 t, 12$ although the period halving is less ideal. This can be

regarded as a pseudo $1 / 4$-periodicity, in which the exact $1 / 4$ period is inhibited due to the incommensurability of $N_{a}$ with 4 .

In Fig.13 we compare the $J_{C}$ obtained from the extended period halving with the phase diagram obtained from the TL analysis by Hayward et al.25 and independently by Sano2 2 . The value of $J_{C}$ falls on a line near the trajectory of $K_{\rho}=1$ and nearly pararell to the boundary between superconducting and phase-separation regions. 
Here we should comment the following. The period is already halved at $J=0$ (the $t$ ladder) at least for the $6 \times 2$ system. 00 This should be related to the Hubbard ladder (the Hubbard model on a double chain), since the $t$ model is identical to the Hubbard model with $U=\infty$. Thus the Hubbard ladder should have the halved period as well for $U=\infty$, while for $U=0$ the extended period is $N_{a} \Phi_{0}$. This implies that a critical point has to exist at which the extended period changes in the Hubbard ladder. A preliminary calculation for a $6 \times 2$-site Hubbard ladder with 4 electrons indeed indicates a halving of the extended period at a finite $U_{c}$. If this is true, we have another example in which the property of the ladder is distinct from the single chain, since the period is always $N_{a} \Phi_{0}$ in the single-chain Hubbard model with $U>0$.

\section{Quarter filling}

At the quarter filling ( $N_{a}$ electrons on a $N_{a} \times 2$ ladder), a peculiar behavior appears, i.e., the period becomes the smallest possible $\Phi_{0}$ irrespective of $J$ and the systems size $(4 \times 2,6 \times 2$ and $8 \times 2$ sites $)$. As an example we show the ground-state spectral flow for a $6 \times 2$-site system with 6 electrons in Fig.14. The period $\Phi_{0}$ is what is expected for the charge-gapped system (e.g., a Mott insulator). In this sense the $\Phi_{0}$ period may come from the commensulability of the filling, although we could not find any characteristic structure in the CDW correlation $\left\langle n_{i, \alpha} n_{j, \beta}\right\rangle$, which is almost flat in these small systems. The period remains the same when we modify the interaction parameters in the region $t<t_{\perp}, J<J_{\perp}$. On the other hand, if we approach to the independent chains in the region $t>t_{\perp}, J>J_{\perp}$, a branch having a longer period comes downwards to cross the lowest $\Phi_{0}$-period line. This is natural because in the limit of $t \gg t_{\perp}, J \gg J_{\perp}$, the extended period for the ground state should be that for the 1D ring with $N / 2$ electrons. 


\section{V. $T-J-J^{\prime}$ MODEL}

Ogata et al have studied 1D $t-J_{-} J^{\prime}$ model,22 where they added a frustrating next nearestneighbor exchange interaction $J^{\prime}$ to the $t-J$ model. Like ladder systems, this has been motivated from an expectation that we can tune the spin gap by introducing $J^{\prime} . J^{\prime}$ is in fact shown to give rise to a spin gap at half-filling, which survives for small doping and arbitrary values of $J$. In this section, we apply our method to $t-J_{-} J^{\prime}$ model to discuss whether the extended $\mathrm{AB}$ period can detect a symptom of changes in characters of quantum state.

In particular, the halving point, $J_{C}$, of the extended $\mathrm{AB}$ period from $N_{a} \Phi_{0} / 2$ to $N_{a} \Phi_{0} / 4$ is of interest. (As in 1D $t-J$ model, the extended AB period halves to $N_{a} \Phi_{0} / 2$ right after the $J$ and $J^{\prime}$ set in.) We fix $J^{\prime} / J=0.5$, because Ogata et a 22 determined the phase diagram for this case. The present result shows that $J_{C}=3.4 t$ for 4 electrons in 8 sites, $J_{C}=2.8 t$ for 4 electrons in 10 sites, and $J_{C}=2.6 t$ for 4 electrons in 12 sites. In Fig.15, we superpose the present result on the phase diagram due to Ogata et a 22 . We can see that $J_{C}$ determined by the extended $\mathrm{AB}$ effect falls near the neighborhood of the boundary of phase separation obtained by the exact diagonalization method.

\section{EXTENDED HUBBARD MODEL}

So far we have studied models for which the phase diagram contains spin-gapped regions. However, we have not studied the behavior of the AB period in such regions so far. It is interesting to investigate how the extended $\mathrm{AB}$ period behaves in the region where spin gap opens, since the opening of a spin gap is regarded as a tendency toward attraction between electrons. In the extended Hubbard model, where we consider the off-site (nearest-neighbor) interaction $V$ in addition to the on-site Hubbard $U$, the phase diagram has both spin-gapped and spin-gapless regions bisected by the so-called Luther-Emery line. Thus the model is ideal for studying how the existence or otherwise of the spin gap affects the extended AB effect.

The extended Hubbard model is defined by the Hamiltonian, 


$$
H=-t \sum_{<i, j>\sigma}\left(c_{i \sigma}^{\dagger} c_{j \sigma}+\text { H.c. }\right)+U \sum_{i} n_{i \uparrow} n_{i \downarrow}+V \sum_{i \sigma} n_{i \sigma} n_{i+1 \sigma} .
$$

According to the weak-coupling theory, the superconducting correlation dominates in the region

$$
U+2\left[2-\cos \left(2 k_{F}\right)\right] V<0
$$

and the spin gap opens in the region

$$
U+2 V \cos \left(2 k_{F}\right)<0
$$

For the strong-coupling region, the phase diagram has to be obtained numerically. Making use of the method same as the 1D $t$ - $J$ study by Ogata et $a \sqrt{18}$, Sano and Ono 23 , and independently three of the present authors 24, have numerically determined the phase diagram.

Here we focus on the region with $V<0$ for which the pairing is expected. When $V$ set in the extended $\mathrm{AB}$ period halves to $N_{a} \Phi_{0} / 2$ regardless of the value of $U$. We have determined the point where the extended AB period is halved from $N_{a} \Phi_{0} / 2$ to $N_{a} \Phi_{0} / 4$ for 4 electrons in 8 sites, 4 electrons in 10 sites, and 4 electrons in 12 sites. In Fig.16, we superpose our results

on the phase diagram due to Sano and Ōno 23 . The halving point of the extended AB period is seen to run roughly parallel to the normal-superconductivity and superconductivity-phase separation boundaries, and falls on the superconductivity region. 1

\section{CONCLUDING REMARKS}

In four types of strongly correlated electron systems in 1D, namely

1. $t$ - $J$ model,

2. $t$ - $J$ ladder,

3. $t-J-J^{\prime}$ model, 
4. extended Hubbard model,

we have obtained the extended AB period both from the Bethe-ansatz analysis where possible, and from numerical calculations. While the extended AB period is the system length times the flux quantum for noninteracting systems, we have found the existence of the boundary across which the period is halved or another boundary into an even shorter period on the phase diagram for these models. If we compare this result with the phase diagram predicted from the Tomonaga-Luttinger theory, the halved period (or shorter periods) has a one-to-one correspondence to the pairing (phase separation or metal-insulator transition) in these models. Namely, when the halved (quarter) AB period is detected, the corresponding TL phase diagram has a paired (phase-separated) region. Such an applicability of the extended $\mathrm{AB}$ test extends to the cases where the conventional anomalous flux quantization gives ambiguous results as is the case with the Hubbard or $t-J$ models.

Surprising aspects of these results are:

1. The extended AB flows involve high-energy states, while the TL theory is only intended for the low-energy physics.

2. The extended AB period does not change across the integrable-nonintegrable boundary despite the totally different level statistics.

An important future problem is how the extended $A B$ analysis can be coupled with Berry's geometrical phase. Korepin and $\mathrm{Wu}^{52}$ have in fact calculated for the XXZ Heisenberg magnet Berry's phase from the Bethe-ansatz solution based on the periodicity $\left(\Phi=2 \Phi_{0}\right)$ found by Sutherland and Shastry. Berry's phase, $\gamma$, is expressed in a standard way as

$$
\gamma=\operatorname{Re}\left[i \int_{-\Phi_{0}}^{\Phi_{0}} d \Phi \frac{\left\langle\psi(\Phi)\left|\frac{\partial}{\partial \Phi}\right| \psi(\Phi)\right\rangle}{\langle\psi(\Phi) \mid \psi(\Phi)\rangle}\right]
$$

We can in principle calculate Berry's phase for electron systems such as the Hubbard model similarly. The geometrical phase will help identifying the nature of the many-body states in a gauge field theoretic manner. 


\section{ACKNOWLEDGMENTS}

We wish to thank Prof. V.E. Korepin for drawing our attention to Ref.52 and Prof. N. Kawakami, Dr. X. Zotos and Dr. H. Asakawa for valuable discussions.

Numerical calculations were performed on FACOM VPP500 in Supercomputer Center, Institute for Solid State Physics, University of Tokyo. This work was supported in part by a Grant-in-Aid for Scientific Research on Priority Areas from the Ministry of Education, Science and Culture, Japan. 


\section{REFERENCES}

${ }^{1}$ N. Byers and C.N. Yang: Phys. Rev. Lett. 7 (1961) 46.

${ }^{2}$ R. M. Fye et al.: Phys. Rev. B 44 (1991) 6909.

${ }^{3}$ E. Dagotto and J. Riera: Phys. Rev. Lett. 70 (1993) 682.

${ }^{4}$ F. Assaard et al.: Phys. Rev. B 50 (1994) 12835.

${ }^{5}$ A. Sudbo et al.: Phys. Rev. Lett 70 (1993) 978.

${ }^{6}$ D. Poiblanc: Phys. Rev. B 44 (1991) 9562.

${ }^{7}$ V. A. Kashurnikov and E. G. Kholmovskii: JETP 83 (1996) 1037.

${ }^{8}$ A. Ferretti I.O. Kulik and A. Lami: Phys. Rev. B 45 (1992) 5486.

${ }^{9}$ B. S. Shastry and B. Sutherland: Phys. Rev. Lett. 65 (1990) 243.

${ }^{10}$ D.J. Scalapino, S.R. White, and S.C. Zhang: Phys. Rev. Lett. 68 (1992) 2830.

${ }^{11}$ D.J. Scalapino, S.R. White, and S.C. Zhang: Phys. Rev. Phys. Rev. B 47 (1993) 7995.

${ }^{12}$ K. Kusakabe and H. Aoki: J. Phys. Soc. Jpn 65 (1996) 2772 .

${ }^{13}$ B. Sutherland: Phys. Rev. Lett 74 (1995) 816.

${ }^{14}$ T. Fukui and N. Kawakami: Phys. Rev. Lett. 76 (1996) 4242.

${ }^{15}$ T. Fukui and N. Kawakami: YITP preprint.

${ }^{16}$ K. Kusakabe and H. Aoki: J. Low. Temp. Phys. 105 (1996) 609 .

${ }^{17}$ M. Di Stasio and X. Zotos: Phys. Rev. Lett. 74 (1995) 2050.

${ }^{18}$ M. Ogata, M.U. Luchini, S. Sorella, and F.F. Assaad: Phys. Rev. Lett. 66 (1991) 2388 .

${ }^{19}$ H.J. Schulz: Phys. Rev. Lett. 64 (1990) 2831.

${ }^{20}$ N. Kawakami and S.-K. Yang: Phys. Lett. A 148, (1990) 359. 
${ }^{21}$ H. Frahm and V.E. Korepin: Phys. Rev. B 42 (1990) 10553 .

${ }^{22}$ M. Ogata, M. U. Luchini and T. M. Rice: Phys. Rev. B 44 (1991) 12083.

${ }^{23}$ K. Sano and Y. Ōno: J. Phys. Soc. Jpn. 63 (1994) 1250.

${ }^{24}$ K. Kuroki, K. Kusakabe and H. Aoki: Phys. Rev. B 50 (1994) 575 .

${ }^{25}$ C.A. Hayward and D. Poilblanc: Phys. Rev. B 53 (1996) 11721.

${ }^{26}$ K. Sano: J. Phys. Soc. Jpn. 65 (1996) 1146.

${ }^{27}$ H. Tsunetsugu, M. Troyer, and T.M. Rice: Phys. Rev. B 51 (1995) 16456.

${ }^{28}$ Note that for $N=4 n$ (n: integer), the energy takes its minimum at $\Phi=0.5 \Phi_{0}$ (i.e. for anti-periodic boundary condition).

${ }^{29}$ E.H. Lieb and F.Y. Wu: Phys. Rev. Lett. 20 (1968) 1445.

${ }^{30}$ M. Ogata and H. Shiba: Phys. Rev. B 41 (1990) 2326.

${ }^{31}$ C.K. Lai: J. Math. Phys. 15 (1974) 1675.

${ }^{32}$ P. Schlottmann: Phys. Rev. B 36 (1987) 5177.

${ }^{33}$ B. Sutherland: Phys. Rev. B 12 (1975) 3795.

${ }^{34}$ P.-A. Bares, J.M.P. Carmelo, J. Ferrer and P. Horsch: Phys. Rev. B 46 (1992) 14624.

35 There is yet another formalism introduced by F.H.L. Essler and V.E. Korepin [Phys. Rev. B 46 (1992) 9147], and independently by A. Foerster and M. Karowski [Nucl. Phys. B396 (1993) 611], where an algebraic Bethe Ansatz is employed.

${ }^{36}$ P.-A. Bares, G. Blatter, and M. Ogata: Phys. Rev. B 44 (1991) 130.

${ }^{37}$ E. Dagotto, T.M. Rice: Science 271 (1996) 618.

${ }^{38}$ E. Dagotto, J. Riera and D. Scalapino: Phys. Rev. B 45 (1992) 5744. 
39 T.M. Rice, S. Gopalan and M. Sigrist: Europhys. Lett. 23 (1993) 445.

40 There are now indications that three-leg ladders also superconduct. See T. Kimura, K. Kuroki and H. Aoki: Phys. Rev. B 54 (1996) R9608

${ }^{41}$ E.M. McCarron, III, M.A. Subramanian, J.C. Calabrese and R.L. Harlow: Mat. Res. Bull. 23 (1988) 1355 .

${ }^{42}$ T. Siegrist, L.F. Schneeneyer, S.A. Sunshine, J.V. Waszczak and R.S. Roth: Mat. Res. Bull. 23 (1988) 1429.

${ }^{43}$ Z. Hiroi, M. Azuma, M. Takano and Y. Bando: J. Solid State Chem. 95 (1991) 230.

${ }^{44}$ M. Uehara, et al.: J. Phys. Soc. Jpn 65 (1996) 2764.

${ }^{45}$ H. Takahashi, et al., preprint.

${ }^{46}$ H. Tsunetsugu, M. Troyer, and T.M. Rice: Phys. Rev. B 49 (1994) 16078.

${ }^{47}$ D. Poilblanc, D.J. Scalapino and W. Hanke: Phys. Rev. B 52 (1995) 6796.

${ }^{48}$ C.A. Hayward, D. Poilblanc. R.M. Noack, D.J. Scalapino and W. Hanke: Phys. Rev. Lett. 75 (1995) 926 .

${ }^{49}$ M. Troyer, H. Tsunetsugu and T.M. Rice: Phys. Rev. B 53 (1996) 251.

${ }^{50}$ For the $8 \times 2$ system we cannot obtain the whole spectral flow at $J=0$ because of a numerical difficulty.

${ }^{51}$ According to the calculation by Sano and Ōno, there is a superconducting and phaseseparation region in the region $V>0$ as well. Our preliminary calculation indicates another transition point from $N_{a} \Phi_{0} / 2$ to $N_{a} \Phi_{0} / 4$ in $V>0$ in qualitative agreement with this result.

${ }^{52}$ V.E. Korepin and A.C.T. Wu: Int. J. Mod. Phys. B 5 (1991) 497. 


\section{FIGURES}

FIG. 1. An Aharonov-Bohm flux pierced in an opening of a ring.

FIG. 2. The extended AB flow for a 10-site Hubbard ring with 6 electrons ${ }^{12}$. The flow lines represent a repulsive interaction $(U>0)$, an attractive interaction $(U<0)$, and the noninteracting case.

FIG. 3. Numerical result for the spectral flow for a $1 \mathrm{D} t-J$ ring with 4 electrons in 8 sites.

FIG. 4. Numerical result for the spectral flow for a 1D $t$ - $J$ ring with 4 electrons in 10 sites.

FIG. 5. Numerical result for the spectral flow for a 1D $t$ - $J$ ring with 4 electrons in 12 sites.

FIG. 6. The rapidities for the hole charge $\left(w_{j}\right.$ : thin lines) and for spin-charge degrees of freedom $\left(v_{\alpha}\right.$ : bold lines) against the flux for the ground state for a $1 \mathrm{D} t$ - $J$ ring with 4 electrons in 10 sites. Tiny gaps in the non-diverging branches correspond to the points where a pair of branches diverge to $\pm \infty$.

FIG. 7. The change of the spectral flow when small $J$ is turned on.

FIG. 8. The motion of charge rapidities for a finite $J$ in $1 \mathrm{D} t-J$ model.

FIG. 9. The present result for the halving points (open circles) of the extended AB period in $1 \mathrm{D} t-J$ model is superposed on the phase diagram obtained by Ogata et $a .^{18}$

FIG. 10. The extended $\mathrm{AB}$ flow for an $8 \times 2$-site $t$ - $J$ ladder with two electrons for $J=0$, $J=0.4 t$ and $J=0.8 t$, respectively.

FIG. 11. The extended $\mathrm{AB}$ flow for an $8 \times 2$-site $t$ - $J$ ladder with four electrons for $J / t=0,2.0,2.6,3.0,3.4$ from top to bottom. 
FIG. 12. The extended $\mathrm{AB}$ flow for an $6 \times 2$-site $t$ - $J$ ladder with four electrons for $J=0,2.0,2.2,2.6, \cdots, 3.8$ from top to bottom

FIG. 13. Halving points, $J_{C}$, of the extended AB period (big crosses) in the $t-J$ ladder are compared with the trajectory of $K_{\rho}=1$ (the normal-superconducting boundary) obtained by the exact diagonalization (solid squares by Hayward and Poilblanc ${ }^{25}$, solid triangles by Sano ${ }^{26}$ ). Phase separation boundary $\left(K_{\rho}=\infty\right)$ are also displayed by open squares or open triangles.

FIG. 14. The extended AB flow for an $6 \times 2$-sites $t$ - $J$ ladder with six electrons (the quarter-filling) for $J=0$ and $J=t$. The period is $\Phi_{0}$ irrespective of $J$.

FIG. 15. The present result for the halving points (filled circles) of the extended AB period in 1D $t-J-J^{\prime}$ model is superposed on the phase diagram obtained by Ogata et $a l .^{22}$

FIG. 16. The halving point of the extended AB period in the extended Hubbard model for four electrons in an 8-site system (band filling $n=0.5$; open circles), 10-site system $(n=0.4$; open triangle), and 12 -site system ( $n=0.333$; open square) is superposed on the phase diagram obtained by Sano et $a l .{ }^{23}$ and Kuroki et $a l .{ }^{24}$ for $n=0.5$. For $n=0.5$, The line $U=0$ is the boundary between the spin-gapped and spin-gapless regions. 